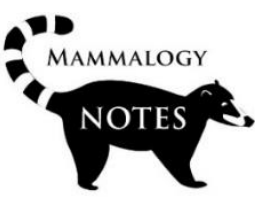

\title{
Consumo de inflorescencia de maguey Agave sp. (Aspargales: Aspargaceae) por la ardilla de Peters Parasciurus oculatus (Rodentia: Sciuridae) en la Reserva de la Biosfera Sierra Gorda de Guanajuato, México
}

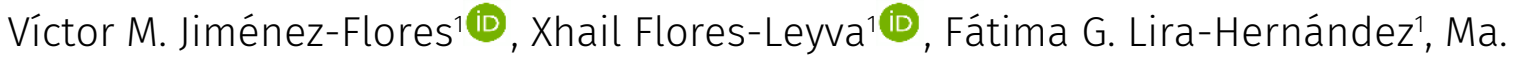 \\ Eugenia Mendiola-González' (iD), Alicia Zárate-Martínez'iD, Luis F. Vázquez- \\ Sandoval', Juan F. Charre-Medellín ${ }^{2,3 *}$ (iD)

\begin{abstract}
1 Reserva de la Biosfera Sierra Gorda de Guanajuato, Comisión Nacional de Áreas Naturales Protegidas, Jiménez 222, Centro, 37900, San Luis de la Paz, Guanajuato, México.

2 Centro de Investigaciones en Geografia Ambiental, Universidad Nacional Autónoma de México, Antigua Carretera A Pátzcuaro 8701, Ex Hacienda San José de la Huerta, 58190, Morelia Michoacán, México.

3 Conservación del Patrimonio Natural para el Bienestar Social A. C., Francisco Mayagoitia Olmos 114, Humanista 1, 36790, Salamanca, Guanajuato, México.
\end{abstract} \\ *Correspondencia: jcharre@ciga.unam.mx
}

\section{Resumen}

Aproximadamente el $87,5 \%$ de las especies de plantas con flores son polinizadas por animales, sin embargo, la magnitud del impacto en la polinización de plantas por parte de los roedores, grupo al que pertenecen las ardillas, ha sido subestimada por la falta de información. Documentamos por primera ocasión consumo de las inflorescencias, néctar o polen de maguey (Agave sp.) por la ardilla de Peters (Parasciurus oculatus). Es necesario continuar el monitoreo de los polinizadores de los magueyes, para determinar el papel que juegan las ardillas en los procesos de polinización o depredación de estas plantas.

Palabras clave: Maguey, néctar, polinización, RBSGG, Sciuridae.

Abstract

Approximately $87,5 \%$ of flowering plant species are pollinated by animals, however, the magnitude of the impact on plant pollination by rodents, a group to which squirrels belong, has been underestimated due to the lack of information. For the first time, we documented the consumption of inflorescences, nectar or pollen of maguey (Agave sp.) by the Peters squirrel (Parasciurus oculatus). It is necessary to continue monitoring the pollinators of the magueys, in order to determine the role that squirrels play in the pollination or predation processes of these plants.

Key words: Maguey, nectar, pollination, SGBRG, Sciuridae. 
La relación planta-animal, particularmente la asociada a la polinización, ha sido documentada ampliamente como mutualista, ya que la mayoría de los visitantes florales obtienen alimento como flores, néctar, polen o aceites, mientras que las plantas aseguran su fecundación (Bascompte et al. 2003; Trejo-Salazar et al. 2015). Se estima que el 87,5 \% de las especies de plantas con flores son polinizadas por animales (Ollerton et al. 2011). Sin embargo, mucha de la información sobre la polinización animal se ha centrado en los insectos, mientras que el papel de los polinizadores vertebrados no ha sido tan ampliamente reconocido más allá de algunos grupos particulares como los murciélagos y aves (Ollerton et al. 2011; Ratto et al. 2018). La magnitud del impacto en la polinización de plantas por parte de los roedores, donde se incluyen las ardillas, ha sido subestimado por la falta de información, particularmente, para el grupo de las ardillas de América (Ratto et al. 2018).

Recientemente se ha evaluado la clasificación taxonómica de las ardillas arborícolas de la familia Sciuridae, donde la ardilla de Peters cambió su nombre de Sciurus oculatus a Parasciurus oculatus (de Abreu-jr et al. 2020). Dentro de las 278 especies de ardillas que habitan alrededor de los cinco continentes y las 35 especies distribuidas en México, se encuentra la ardilla de Peters ( $P$. oculatus Peters, 1863), la cual es una especie endémica y considerada bajo protección especial de acuerdo a las normas oficiales mexicanas, distribuyéndose principalmente en los bosques templados del centro del país, entre los 1.500 y 3.600 msnm (Hall 1981; Best 1995; Valdéz-Alarcón \& Téllez-Giron 2005; Thorington \& Ferrell 2006; SEMARNAT 2010; Monterrubio-Rico et al. 2013). La dieta de la ardilla de Peters se basa principalmente en bellotas, conos de pinos, semillas, frutos y polen de Oyameles (Abies religiosa) (Best 1995; Valdéz-Alarcón \& Téllez-Giron 2005; Ramos-Lara \& LópezGonzález 2017). No obstante, hasta el momento no se había documentado como parte de la dieta de las ardillas arborícolas de la familia Sciuridae las inflorescencias, néctar o polen de los magueyes (Agave spp.), ni el potencial de estos roedores en la polinización de este grupo de plantas de importancia ecológica, económica y cultural para México.

Los magueyes son uno de los grupos de plantas más diversos en México, con alrededor de 280 especies, los cuales producen inflorescencias que constituyen un importante recurso para los visitantes florales que participan en su proceso de polinización (Trejo-Salazar et al. 2015). Se ha documentado que los principales visitantes florales y potenciales polinizadores de los magueyes son los murciélagos, esfingidos, abejas, avispas, abejorros, lepidópteros y aves (colibries y aves percheras) (Trejo-Salazar et al. 2015). Por tal motivo, el objetivo de este trabajo es documentar por primera vez el consumo de inflorescencias, néctar o polen de magueyes por parte de la ardilla de Peters y su potencial importancia en la polinización o depredación de dichas especies.

La Reserva de la Biosfera Sierra Gorda de Guanajuato (RBSGG), es un área natural protegida de carácter federal que se localiza en el centro de México, abarcando cinco municipios del noreste del estado de Guanajuato (Figura 1) (SEMARNAT 2007). De las 236.882 hectáreas con las que cuenta la RBSGG, el 87 \% corresponde a vegetación natural de la cual el 39 \% son matorrales, 37 \% bosques templados y 11 \% selva baja caducifolia (Pérez-Vega et al. 2016). La RBSGG presenta una enorme heterogeneidad ambiental debido a su gradiente altitudinal, que va desde los 650 a 2.600 msnm, con climas semiáridos, cálidos subhúmedos y templados, lo que permite que sea una región en el centro del país con alta biodiversidad (Cuevas \& Zorrilla 2014). En la RBSGG se presentan temperaturas mínimas promedio de $10^{\circ} \mathrm{C}$ y máximas de $33^{\circ} \mathrm{C}$, mientras que la precipitación oscila entre los 400 y 1.200 mm anualmente (Martínez-Arredondo et al. 2013). 
Como parte de los programas de monitoreo de biodiversidad realizados por el personal de la RBSGG, y particularmente el monitoreo del éxito reproductivo del Águila real (Aquila chrysaetos) que se realiza de manera continua en la RBSGG desde el año 2.018, el 26 de mayo del 2.020 se observó a una ardilla de Peters consumiendo inflorescencias, néctar o polen de maguey (Agave sp.) (Figura 2A). El evento ocurrió en el paraje de la Mesa de los caballos (21,324068, -100,345403, WGS84), en la comunidad Mesita del Tigre, en el municipio de Victoria, Guanajuato, dentro de la RBSGG (Figura 1 y 2). El sitio de observación se ubica a 2.230 msnm, en una zona de matorral xerófilo con fragmentos de bosque de encino, donde la presencia de magueyes al igual que la de ardillas de Peters, es abundante. El evento ocurrió a las 16 h52 y tuvo una duración de 12 minutos. El maguey pulquero tenía una inflorescencia con una espiga de $4 \mathrm{~m}$ de altura (Figura 2B). Debido a la simpatría que existe en las ardillas arboricolas ( $P$. oculatus y Echinosciurus aureogaster) en el centro de México (Ramos-Lara \& López-González 2017), se muestran los registros de estas dos especies de ardillas arborícolas y registros de magueyes reportados en la RBSGG (Figura 1). Estos registros provienen de la base de datos recopilada de diversas fuentes bibliográficas por parte del personal de RBSGG.
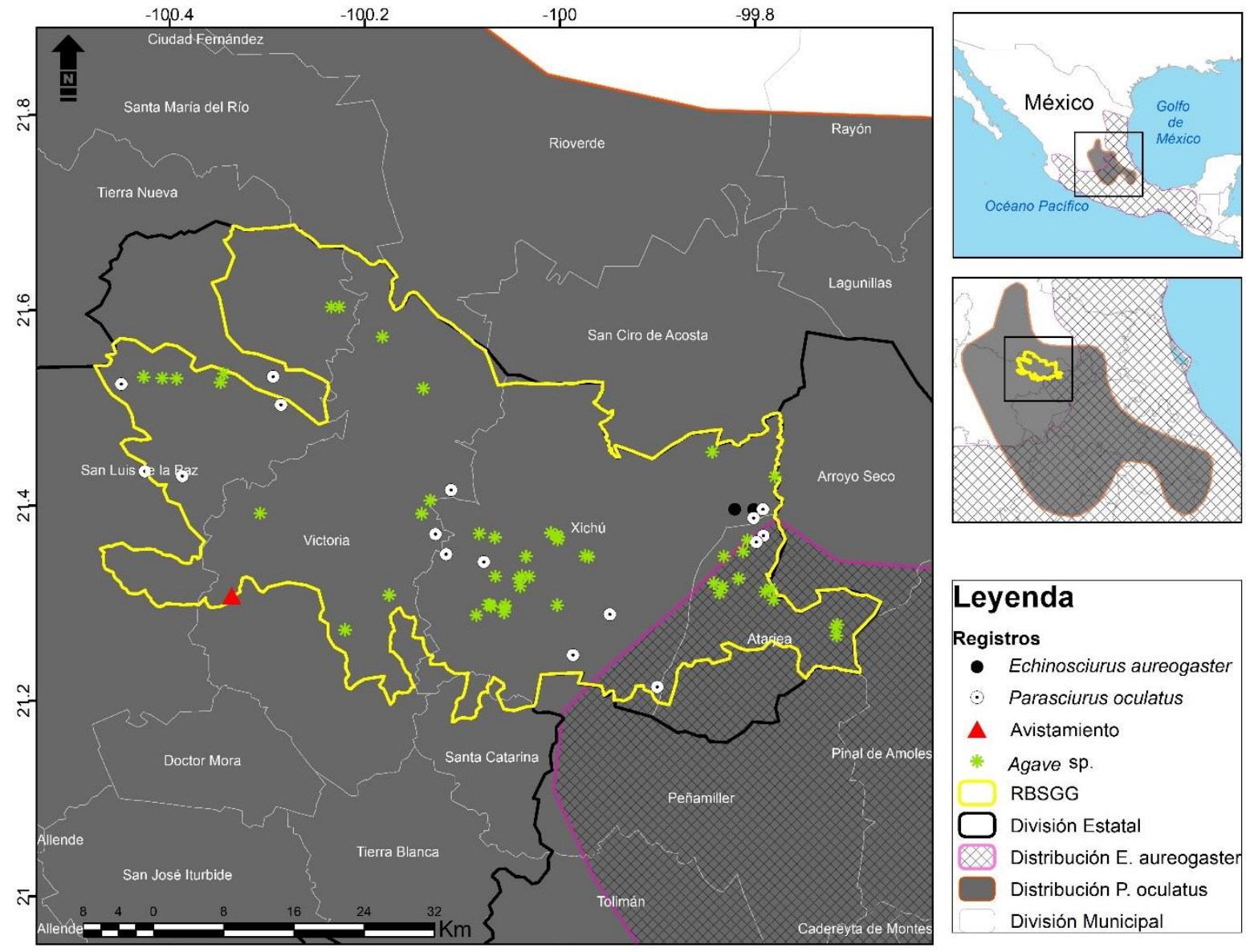

\section{Leyenda}

Registros

- Echinosciurus aureogaster

- Parasciurus oculatus

$\Delta$ Avistamiento

Agave sp. RBSGG

División Estatal

Distribución E. aureogaster Distribución P. oculatus División Municipal

FIGURA 1. Ubicación del avistamiento de la ardilla de Peters ( $P$. oculatus) alimentándose de la inflorescencia del maguey (Agave sp.) (triangulo rojo). Se señalan los registros tanto de P. oculatus y E. aureogaster, como de magueyes (Agave spp.) reportados para la RBSGG. 

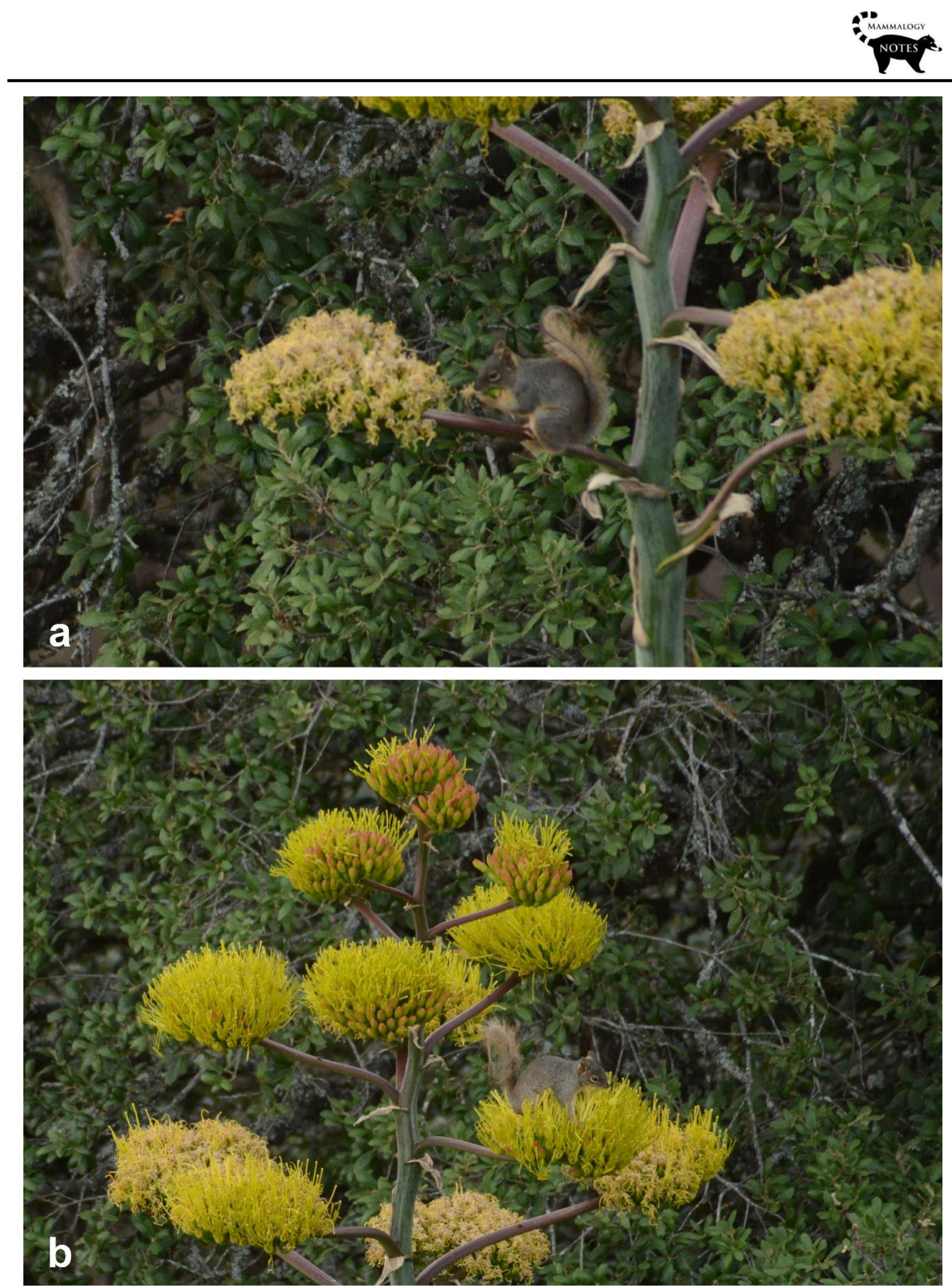

FIGURA 2. Ardilla de Peters (P. oculatus) alimentándose de inflorescencias del maguey (Agave sp.) en la RBSGG. a) Ardilla consumiendo inflorescencia de maguey. b) Ardilla sobre la inflorescencia de maguey. 
Las inflorescencias, néctar o polen de maguey se agregan a la dieta de la ardilla de Peters reportada anteriormente, donde no se menciona el consumo de estos elementos en su dieta (Best 1995; Valdéz-Alarcón \& Téllez-Giron 2005; Monterrubio-Rico et al. 2013; RamosLara \& López-González 2017). En general, la dieta de las ardillas puede variar a lo largo del año con base en la disponibilidad de recursos. Para algunas especies de ardillas arboricolas como P. niger y E. aureogaster, o la ardilla voladora Glaucomys volans, se ha documentado que durante la primavera se alimentan de una gran variedad de brotes y flores de plantas, cambiando su dieta durante el verano a insectos, mientras que, durante el otoño e inverno, las nueces, semillas y piñas de los pinos son los elementos más importantes en su alimentación (Thorington \& Ferrell 2006; Ramos-Lara \& López-González 2017). Es probable que este mismo fenómeno ocurra con la ardilla de Peters en sitios altamente heterogéneos, como la RBSGG, donde la disponibilidad de recursos puede variar a lo largo del año.

Además de la incorporación de nuevos elementos a la dieta de la ardilla de Peters, es importante evaluar el potencial que pueden tener los mamíferos no voladores en el proceso de polinización o consumo de las inflorescencias de los magueyes, como se ha observado por parte de los Coatís (Nasua narica) en Arizona y cuya especie también se encuentra presente en la RBSGG (McColgin et al. 2003). En otras regiones del mundo se ha documentado la importancia de las ardillas en la polinización de diversas plantas, debido a que el polen queda adherido a los dedos, boca y pelaje de las ardillas, de tal manera que al caminar sobre la estructura de la inflorescencia podrían dispersar el polen entre las flores de diferentes individuos de maguey (Yumoto et al. 2000; Kobayashi et al. 2017; Palacios-Mosquera et al. 2019). Sin embargo, también se ha reportado que la ardilla $E$. aureogaster, especie simpátrica de la ardilla de Peters, es responsable del consumo de las flores de una bromelia en bosques mesófilos, actuando como depredadores florales, afectando hasta un 63 \% de las plantas, lo cual puede tener implicaciones en la reproducción de la planta (García-Franco \& Rico-Gray 1991).

Recientemente se ha enfatizado sobre la necesidad de conservar los polinizadores vertebrados debido a su valiosa participación en la polinización de plantas cultivadas de importancia económica como los magueyes (Ratto et al. 2018). Es por ello que es importante conservar aquellas especies consideradas raras o frágiles, como la ardilla de Peters. Especie para la que la tala y la fragmentación de los bosques son los factores más severos que ponen en riesgo sus poblaciones a largo plazo. Por tal motivo, se le ha considerado dentro de las especies de alta prioridad para su conservación en el estado de Guanajuato (Valdéz-Alarcón \& Téllez-Giron 2005; Sánchez et al. 2016; Ramos-Lara \& López-González 2017). Es necesario continuar el monitoreo de la biodiversidad en la RBSGG, y particularmente el de los visitantes florales de aquellas especies de plantas con valor ecológico, económico y cultural como los magueyes, a fin de determinar el papel que juegan los vertebrados, en los procesos de polinización o depredación de estas plantas.

\section{AGRADECIMIENTOS}

A FAUNISTICA CIERE, A.C. por los recursos financieros para el monitoreo de águila real. A la Comisión Nacional de Áreas Naturales Protegidas (CONANP) por financiar parcialmente esta investigación. Al FMCN por el convenio de colaboración con FAUNISTICA CIERE, A. C. Charre-Medellín agradece al CONACYT por la beca otorgada (239248) y a la Dirección General de Asuntos del Personal Académico (DGAPA), UNAM por la beca posdoctoral otorgada. 


\section{REFERENCIAS}

Bascompte J, Jordano P, Melián CJ, Olesen JM. 2003. The nested assembly of plant-animal mutualistic networks. PNAS 100(16):9383-9387. https:// doi.org/10.1073/pnas.1633576100

Best T. 1995. Sciurus oculatus. Mammilan Species 498:1-3. https:// doi.org/10.1644/0.498.1

Cuevas J, Zorrilla M. 2014. Localización y Superficie. In: (CONABIO). Comisión Nacional para el Conocimiento y Uso de la Biodiversidad, En: La Biodivers en Guanajuato Estud Estado Vol I. México: Comisión Nacional para el conocimiento y uso de la Biodiversidad, Instituto de Ecología de Guanajuato; p. 28-37.

de Abreu-jr F, Pavan SE, Tsuchiya MTN, Wilson DE, Percequillo AR, Maldonado JE. 2020. Museomics of tree squirrels: a dense taxon sampling of mitogenomes reveals hidden diversity, phenotypic convergence, and the need of a taxonomic overhaul. Research Square 20:1-25. https:// doi:10.21203/rs.3.rs-28614/v1

García-Franco J, Rico-Gray V. 1991. Biología reproductiva de Tillandsia deppeana Steudel (Bromeliaceae) en Veracruz, México. Brenesia 35:61-79.

Hall E. 1981. The mammals of North America. New York, E.U.A.: John Wiley and Sons.

Kobayashi S, Denda T, Liao C, Wu S, Lin Y, Izawa M. 2017. Squirrel pollination of Mucuna macrocarpa (Fabaceae) in Taiwan. Journal of Mammalogy 98(2):533-541. https:// doi.org/10.1093/jmammal/gyw189

Martínez-Arredondo J, Ortega-Chávez V, Ramos-Arroyo R. 2013. Índices climatológicos regionales para la Sierra Gorda de Guanajuato. Acta Universitaria 23(6):10-25.

McColgin M, Brown E, Bickford S, Eilers A, Koprowski J. 2003. Use of century plants (Agave palmeri) by coatis (Nasua narica). Southwest Naturalist 48(4):722-725. https:// doi.org/10.1894/00384909(2003)048<0722:UOCPAP>2.0.CO;2

Monterrubio-Rico T, Guido-Lemus D, Charre-Medellín J, Zavala-Paramo M, Padilla-Jacobo G, CanoCamacho H, Leon-Paniagua L. 2013. Nuevos registros de la ardilla de Peters Sciurus oculatus (Peters 1863) para Michoacán y primera genotipificación molecular de la especie. Acta Zoológica Mexicana 29(2):304-316.

Ollerton J, Winfree R, Tarrant S. 2011. How many flowering plants are pollinated by animals? Oikos 120:321-326.

Palacios-Mosquera Y, Mondragón D, Santos-Moreno A. 2019. Vertebrate florivory of vascular epiphytes: the case of a bromeliad. Brazilian Journal of Biology 79(2):201-208.

Pérez-Vega A, Rocha-Álvarez F, Regil-García H. 2016. Distribución espacial del uso cubierta del suelo y degradación forestal en la reserva de la biosfera Sierra Gorda de Guanajuato. Acta Universitaria 26:33-44. https://doi.org/10.15174/au.2016.1500

Ramos-Lara N, López-González C. 2017. Niche segregation between Sciurus aureogaster and S. oculatus in a disturbed forest in central Mexico. Journal of Mammalogy 98(6):1780-1790. https://doi.org/10.1093/jmammal/gyx136

Ratto F, Simmons BI, Spake R, Zamora-Gutierrez V, Macdonald M, Merriman J, Tremlett C, Poppy G, Peh K, Dicks D. 2018. Global importance of vertebrate pollinators for plant reproductive success: a meta-analysis. Frontiers in Ecology and the Environment 16(2):82-90. https:// doi.org/10.1002/fee.1763 
Sánchez O, Charre-Medellín J, Téllez-Girón G, Báez-Montes O, Magaña-Cota G. 2016. Mamíferos silvestres de Guanajuato: actualización taxonómica y diagnóstico de conservación. En: Briones-Salas M, Hortelano-Moncada Y, Magaña-Cota G, Sánchez-Rojas G, Sosa-Escalante J, editors. Riqueza y Conservación los Mamíferos en México a Nivel Estatal. México: Instituto de Biología, Universidad Nacional Autónoma de México, Asociación Mexicana de Mastozoología A. C. y Universidad de Guanajuato; p. 243-280.

SEMARNAT (Secretaría de Medio Ambiente y Recursos Naturales). 2007. Decreto por el que se declara área natural protegida, con el carácter de reserva de la Biósfera, la zona conocida como Sierra Gorda de Guanajuato. México.

SEMARNAT (Secretaría de Medio Ambiente y Recursos Naturales). 2010. Norma Oficial Mexicana (NOM-059-SEMARNAT-2010) Protección ambiental-Especies nativas de México de flora y fauna silvestres-Categorías de riesgo y especificaciones para su inclusión, exclusión o cambio-Lista de especies en riesgo. Ciudad de México, México.

Thorington R, Ferrell K. 2006. Squirrels. The animal answer guide. Baltimore, Maryland, USA: JHU press.

Trejo-Salazar R, Scheinvar E, Eguiarte L. 2015. ¿Quién poliniza realmente los agaves? Diversidad de visitantes florales en 3 especies de Agave (Agavoideae: Asparagaceae). Revista Mexicana de Biodiversidad 86(2):358-369. https://doi.org/10.1016/j.rmb.2015.04.007

Valdéz-Alarcón M, Téllez-Giron G. 2005. Sciurus oculatus Peters, 1863. In: Ceballos G, Oliva G, En: Los mamíferos silvestres México. México: Fondo de Cultura Económica, CONABIO; p. 556-557.

Yumoto T, Kuniyasu M, Nagamasu H. 2000. A New Pollination Syndrome - Squirrel Pollination in a Tropical Rainforest in Lambir Hills National Park, Sarawak, Malaysia. Tropics 9(2):147-151. https:// doi.org/10.3759/tropics.9.147

Editor: José F. González-Maya

Recibido 2020-07-10

Revisado: 2020-07-28

Aceptado 2020-08-05

Publicado 2020-10-09 